\title{
RESIDUALLY SOLVABLE EXTENSIONS OF AN INFINITE DIMENSIONAL FILIFORM LEIBNIZ ALGEBRA
}

\author{
K.K. ABDURASULOV ${ }^{1,3}$, B.A. OMIROV ${ }^{1,3}$, I.S. RAKHIMOV ${ }^{2,3}$, G.O. SOLIJANOVA ${ }^{3}$
}

\begin{abstract}
In the paper we describe the class of all solvable extensions of an infinite-dimensional filiform Leibniz algebra. The filiform Leibniz algebra is taken as a maximal pro-nilpotent ideal of a residually solvable Leibniz algebra. It is proved that the second cohomology group of the extension is trivial.
\end{abstract}

\section{INTRODUCTION}

Lie algebras play an essential role in Mathematics and Physics. Their structure theory has been intensely studied for many years by mathematicians and theoretical physicists. In this paper we will be treating infinite-dimensional Lie and Leibniz algebras. The motivation comes from the results with applications of the solvable extension method to finite-dimensional Lie algebras obtained earlier (see Section (1.1) and finite-dimensional Leibniz algebras obtained recently (see Section 1.3). All algebras considered in the paper are supposed to be over the field of complex numbers $\mathbb{C}$ unless otherwise specified.

1.1. Finite-dimensional Lie algebras. It is well-known that the fundamental Levi theorem stating that every finite-dimensional Lie algebra is isomorphic to the semidirect sum of a semisimple Lie algebra and the maximal solvable ideal (the radical) greatly simplifies understanding the structure of a given Lie algebra. The classification of simple and semisimple Lie algebras were obtained by W. Killing and É. Cartan using root systems. Simple Lie algebras are given as five exceptional algebras $E_{6}, E_{7}, E_{8}, F_{4}$, $G_{2}$ and series $A_{n}, B_{n}, C_{n}$ and $D_{n}$. While a complete classification of solvable and nilpotent Lie algebras seems not to be feasible. As for solvable Lie algebras, then various methods of their classification in low-dimensional cases have been implemented and lists of isomorphism classes were presented. Here are just a few words on one of these methods closely related to that problems studied in this paper. This is a method originated by V.V. Morozov [24] on construction of solvable Lie algebras by their nilradicals. It turned out what was noticed by V.V. Morozov and C.M. Mubarakzjanov that there is interrelations between a few invariants of Lie algebras: the dimension and the number of generators of the nilradical, the co-dimension of the nilradical, the number of nil-independent derivations, the existence of inner and outer derivations, the dimensions of the first and second (co)homology spaces. One of such kind relations states that the co-dimension of the nilradical of the Lie algebra is at most the number its nill-independent derivations. The fact has been used to construct the solvable Lie algebras in 25, 26, 27, 28. By using these relationships the classification of solvable extensions has been given for the following classes of nilpotent Lie algebras in low-dimensions with Abelian [29, 30, Heisenberg [33, Borel 41, $\mathbb{N}$-graded 9, filiform and quasifiliform nilradicals 39, 40, (also see [38, and references therein). A few other nilradicals cases are given in [34, 35, 36]. In the finite-dimensional case, the concept of filiform Lie algebra has been introduced by Vergne in [43. It turned out that the case of filiform and quasi-filiform nilradicals the relationships mentioned above much simplify the situation. Moreover, there are cases when the co-dimension of the nilradical is equal to the number of its generators. In these cases the structure of solvable Lie algebras is quite rigid. It was observed that a such Lie algebra is unique up to isomorphism (see [2]), its center is trivial and all derivations are inner. In addition, often such Lie algebras have trivial second Chevalley cohomology groups (see [4, 20]).

1.2. Infinite-dimensional Lie algebras. As mentioned above the problem of classification of simple finite-dimensional Lie algebras over the field of complex numbers was solved by the end of the 19th

2010 Mathematics Subject Classification. 17B40, 17B56, $17 \mathrm{~B} 65$.

Key words and phrases. Lie algebra, potentially nilpotent Lie algebra, pro-nilpotent Lie algebra, cohomology group. 
century by W. Killing and É. Cartan. And just over a decade later, É. Cartan classified simple infinitedimensional Lie algebras of vector fields on a finite-dimensional space. Then B. Weisfeiler 42 finds an algebraic proof of Cartan's classification theorem reducing the problem to the classification of simple $\mathbb{Z}$-graded Lie algebras of finite "depth".

At the present time there is no general theory of infinite-dimensional Lie algebras. There are, however, four classes of infinite-dimensional Lie algebras that underwent a more or less intensive study due to their various applications mostly in Physics. These are, first of all, the above-mentioned Lie algebras of vector fields, the second class consists of Lie algebras of smooth mappings of a given manifold into a finite-dimensional Lie algebra, the third class is the classical Lie algebras of operators in a Hilbert or Banach space and finally, the fourth class of infinite-dimensional Lie algebras is the class of the so-called Kac-Moody algebras.

In the classification theory of infinite-dimensional Lie algebras, several deep results were obtained with Galois cohomology methods exhibiting exciting connections between forms of multi-loop algebras and the Galois theory of forms of algebras over rings. This branch of structure theory is complemented by the connection between the classification of generalized Kac-Moody algebras and automorphic forms. At the same time, the study of some classes of infinite-dimensional Lie algebras is still in its infancy. Even such concepts as solvability and nilpotency, as well as their interrelations, are not completely understood.

It is known that there is no analogue of the Levi decomposition in infinite-dimensional Lie algebras. Moreover, the analogue of Engel's theorem for infinite-dimensional Lie algebras is also wrong. Only in the 1990s E.Zelmanov could solve the Burnside problem, which connects the nilpotency property of a Lie algebra with adjoint operators satisfying the $n$-Engel's condition [44].

Note that there are some examples of the so-called pro-solvable Lie algebras whose maximal pronilpotent ideal is $\mathbb{N}$-graded Lie algebra of maximal class (infinite-dimensional filiform Lie algebra) the method described above for finite-dimensional solvable Lie algebras by means of its nilradical is applicable (see [3]). It should be noted that in all of these mentioned examples, the codimension of the maximal pro-nilpotent ideal of a pro-solvable algebra coincides with the number of generators of the pro-nilpotent ideal.

The infinite-dimensional analogue of filiform Lie algebras has been introduced by A.Fialowski a long time ago in 11. Nevertheless, the systematic study of infinite-dimensional cases has not been of interest. The attempts were occasional depending mainly on some applications in Physics and Geometry. For instance, in 15, two classes of infinite-dimensional Lie algebras called potentially nilpotent and potentially solvable were introduced in connection with the study of their deformations.

1.3. Finite-dimensional Leibniz algebras. Many results of the theory of Lie algebras have been extended to the case of Leibniz algebras. For instance, the classical results on Cartan subalgebras, Levi's decomposition, properties of solvable algebras with a given nilradical and others from the theory of Lie algebras are also true for Leibniz algebras. D. Barnes [5] has proved an analogue of Levi Theorem for Leibniz algebras. It was shown that a Leibniz algebra is decomposed into a semidirect sum of its solvable radical and a semisimple Lie algebra. Therefore, the description of finite-dimensional Leibniz algebras is reduced to the study of solvable Leibniz algebras. D.Barnes also noted that the nonuniqueness of the semisimple subalgebra $S$ appeared in Levi-Malcev theorem (the minimum dimension of Leibniz algebra where this phenomena appears is six). It is known that in the case of Lie algebras the semisimple Levi quotient is unique up to a conjugation via an inner automorphism. However, the conjugacy property in the case of Leibniz algebras is not true, in general. This phenomenon has been studied in [16]. The authors give the conditions for the semisimple part of Leibniz algebras in the decomposition to be conjugated.

The generalizations of the solvable extension method mentioned in Section 1.1 to some cases of Leibniz algebras have been given in [7, 10, 14, 17, 18. The construction of solvable extensions has been treated for the following classes of Leibniz algebras: with Abelian [13, 18, Heisenberg [6, filiform and null-filiform nilradicals [7, 32, naturally graded filiform [8, 19, naturally graded quasi-filiform [21, the direct sum of null-filiform [17, 18, (also see [37] and references therein). Note that in the paper [21, the construction of a finite dimensional complete Leibniz algebra with the subspace complementary space to the nilradical is not maximal was given. It also was proven there that the second (co)homology group of these type of Leibniz algebras is trivial.

1.4. Infinite-dimensional Leibniz algebras. An extensive study of Lie and Leibniz algebras leaded many beautiful results and generalizations. However, in spite of great interest and applications the 
study of some classes of infinite-dimensional Leibniz algebras is still in its infancy. Such in the case of infinite-dimensional Leibniz algebras the concepts of solvability and nilpotency, as well as their interrelations, are not yet completely understood.

For most classes of the infinite-dimensional Leibniz algebras an analogue of the Levi decomposition and Engel's theorem also do not hold. In this paper we are going to treat infinite-dimensional Lie and Leibniz algebras around the solvable extension method narrated in Sections 1.1 and 1.3. This motivates to introduce a reasonable analogue of the concepts of solvability and nilpotency in the infinitedimensional cases. They have been called pro-nilpotent and pro-solvable algebras. The concept of pronilpotency is defined by two conditions: the intersection of all members of the lower central series is trivial (so-called residually nilpotency property), and the quotient algebra by any member of the central series is finite-dimensional (this condition allows one to endow an infinite-dimensional pro-nilpotent algebra with the topology of the inverse limit of finite-dimensional spaces). One can call "tends to zero" such behaviour of an ideal only with a fair amount of fantasy, for instance, a free finitely generated Leibniz algebra is also pro-nilpotent. Nevertheless, the condition of residual nilpotency is such that finite-dimensional nilpotent Leibniz algebras are also pro-nilpotent. By analogue with solvable and pro-nilpotent Lie (Leibniz) algebras, we consider pro-solvable algebras.

Note that there exist some examples of pro-solvable Lie algebras whose maximal pro-nilpotent ideal is $\mathbb{N}$-graded Lie algebra of maximal class (infinite-dimensional filiform Lie algebra) for which the method of describing finite-dimensional solvable Lie algebras by means of its nilradical is completely agrees. It should be noted that in this case the phenomena occurred in finite-dimensional case, where the dimension of complementary subspace equals the number of generators of the nilradical are valid. That is the structure of infinite-dimensional solvable algebras such that the dimension of the complementary subspace to the nilradical is equal to the number of generators of the nilradical is quite rigid. Such algebras are unique up to isomorphism, their center is zero, and all the derivations are inner. In addition, often such infinite-dimensional algebras have trivial second cohomology groups. Therefore, one of the main goals of the paper is: to determine, in the infinite-dimensional case, analogues of the concepts of solvability and nilpotency; then to extend the method of constructing solvable finite-dimensional Lie and Leibniz algebras in terms of the structure of the nilradical and their nill-independent derivations. Remind that the infinite-dimensional analogue of finite-dimensional filiform Leibniz algebras has been introduced in 31. The author called them thin Leibniz algebras.

The organization of the paper is as follows. In Section 2 we recall some theorems on finitedimensional Lie and Leibniz algebras, definitions needed and some important properties of infinitedimensional Lie and Leibniz algebras. We start Section 3 with studying infinite-dimensional Lie algebras. All residually solvable extensions of the family whose the dimension of the complementary space to maximal pro-nilpotent ideal is less than maximal is classified and it is showed that all the obtained algebras have outer derivations. The completeness of maximal residually solvable Lie algebra with maximal pro-nilpotent ideal $\mathfrak{m}_{0}$ are and trivialness of their second cohomology groups are proven. Section 3.2 is devoted to the study of infinite-dimensional Leibniz algebras. All residually solvable extensions whose maximal pro-nilpotent ideal is an infinite-dimensional analogue of a filiform Leibniz algebra are classified. The triviality of the first and second cohomology groups of the family of the maximal residually solvable extensions are proven. Here one of the differences among the others between Lie and Leibniz algebras appears. Furthermore, in this subsection we provide an example of residually solvable complete Leibniz algebra with not maximal co-dimension (see Theorem 3.9).

The infinite-dimensional algebras considered are supposed to have a countable basis such that any element of the algebra is represented as a finite linear combination of the basis elements.

\section{Preliminaries}

The purpose of this section is to establish the notation, clarify the definitions used in the following text and to give preliminary results.

Definition 2.1. An algebra $L$ over a field $\mathbb{F}$ is said to be a Leibniz algebra if the following identity

$$
[x,[y, z]]=[[x, y], z]-[[x, z], y]
$$

holds true for any $x, y, z \in L$, where $[\cdot, \cdot]$ stands for the product in $L$.

We introduce the notation $\operatorname{Leib}(a, b, c)=[a,[b, c]]-[[a, b], c]+[[a, c], b]$ to use it later.

If $[x, x]=0$ then $\operatorname{Leib}(x, y, z)=0$ is the Jacobi identity. Therefore, a Leibniz algebra is a "noncommutative" analogue of a Lie algebra. 
For a given Leibniz algebra $L$ we define the following two-sided ideals

- $\operatorname{Ann}_{r}(L)=\{x \in L \mid[y, x]=0$, for all $y \in L\}$,

- Center $(L)=\{x \in L \mid[x, y]=[y, x]=0$, for all $y \in L\}$.

called the right annihilator and the center of $L$, respectively.

Definition 2.2. A linear map $d: L \rightarrow L$ is said to be a derivation if for all $x, y \in L$, the following derivation rule

holds true.

$$
d([x, y])=[d(x), y]+[x, d(y)]
$$

The set of all derivations of $L$ is a Lie algebra with respect to the composition. It is denoted by $\operatorname{Der}(L)$. For $x \in L$, as usual, $a d_{x}$ denotes the map $a d_{x}: L \rightarrow L$ defined by $a d_{x}(y)=[x, y], \forall y \in L$. Obviously, $a d_{x}$ is a derivation called inner derivations. The set of all inner derivations of $L$ is denoted by Inner $(L)$. No inner derivations in $\operatorname{Der}(L)$ are called outer derivations. The set Inner $(L)$ is an ideal of $\operatorname{Der}(L)$.

Definition 2.3. A Leibniz algebra $L$ is said to be complete if $\operatorname{Center}(L)=0$ and all its derivations are inner.

Let $R$ be a finite dimensional solvable Leibniz algebra with nilradical $N$ and $Q$ be the subspace complementary to the nilradical $N$. We denote by $N_{\max }$ the class of all algebras with a property that $\operatorname{dim} Q=\operatorname{dim}\left(N / N^{2}\right)$ for some solvable Leibniz algebra $R$. In [12] the following theorem was proven.

Theorem 2.4. Let $R^{\prime}=N \oplus Q^{\prime}$ be a solvable Leibniz algebra such that $N \in N_{\max }$ and $\operatorname{dim} Q^{\prime}<$ $\operatorname{dim}\left(N / N^{2}\right)$. Then $R^{\prime}$ admits an outer derivation.

Let $L$ be an infinite-dimensional Lie (respectively, Leibniz) algebra with countable basis. As usual

$$
L^{1}=L, \quad L^{k+1}=\left[L^{k}, L\right], k \geq 1 ; \quad L^{[1]}=L, \quad L^{[s+1]}=\left[L^{[s]}, L^{[s]}\right], s \geq 1 .
$$

are the lower central and the derived series, respectively. We imitate a definition given in 23. from Lie to Leibniz algebras as follows.

Definition 2.5. A Leibniz algebra $L$ is called residually nilpotent (respectively, solvable) if $\bigcap_{i=1}^{\infty} L^{i}=0$ (respectively, $\left.\bigcap_{i=1}^{\infty} L^{[i]}=0\right)$.

Here are some examples.

Example 2.6. Lie algebra $L$ given on a basis $\left\{e_{0}, e_{1}, e_{2}, \ldots\right\}$ by

$$
L:\left\{\left[e_{0}, e_{i}\right]=e_{i-1}, \quad i \geq 3,\right.
$$

is residually solvable but not residually nilpotent.

Example 2.7. Let $L$ be a Lie algebra given on a basis $\left\{e_{0}, e_{1}, e_{2}, \ldots\right\}$ by the following commutation relations

$$
L:\left[e_{i}, e_{j}\right]=e_{0} .
$$

Then $L$ is both residually nilpotent and residually solvable.

The concepts of potentially nilpotency and solvability also can be extended from Lie algebras case given in [15] to Leibniz algebras as follows.

Definition 2.8. A Leibniz algebra $L$ is said to be potentially nilpotent (respectively, solvable), if $\bigcap_{i=1}^{\infty} L^{i}=0$ (respectively, $\left.\bigcap_{i=1}^{\infty} L^{[i]}=0\right)$ and $\operatorname{dim}\left(L^{i} / L^{i+1}\right)<\infty\left(\right.$ respectively, $\left.\operatorname{dim}\left(L^{[i]} / L^{[i+1]}\right)<\infty\right)$ for any $i \geq 1$.

An infinite-dimensional Leibniz algebra $F$ given on a basis $\left\{e_{1}, e_{2}, \ldots\right\}$ by $\left[e_{i}, e_{1}\right]=e_{i+1} \quad i \geq 2$ is potentially nilpotent but not potentially solvable.

The definitions of pro-nilpotency and pro-solvability of algebras have been introduced in [22] as follows.

Definition 2.9. An algebra $L$ is called pro-nilpotent (respectively, pro-solvable), if $\bigcap_{i=1}^{\infty} L^{i}=0$ and $\operatorname{dim}\left(L / L^{i}\right)<\infty$ (respectively, if $\bigcap_{i=1}^{\infty} L^{[i]}=0$ and $\left.\operatorname{dim}\left(L / L^{[i]}\right)<\infty\right)$ for any $i \geq 1$. 
Observe that there is the following isomorphism of vector spaces:

$$
L / L^{i} \cong L / L^{2} \oplus L^{2} / L^{3} \oplus \cdots \oplus L^{i-1} / L^{i}
$$

which implies

$$
\operatorname{dim}\left(L / L^{i}\right)=\sum_{k=1}^{i-1} \operatorname{dim}\left(L^{k} / L^{k+1}\right)<\infty .
$$

This means that the definitions of potentially nilpotency of Lie (respectively, Leibniz) algebras and pro-nilpotency of Lie (respectively, Leibniz) algebras are equivalent.

The same arguments due to the isomorphisms of vector spaces

$$
L / L^{[i]} \cong L / L^{[2]} \oplus L^{[2]} / L^{[3]} \oplus \cdots \oplus L^{[i-1]} / L^{[i]}
$$

assure that potentially solvable Lie (respectively, Leibniz) algebra is a pro-solvable Lie (respectively, Leibniz) algebra and vice versa, i.e., Definitions 2.8 and 2.9 the above are equivalent.

Note that the quotients $L / L^{i}$ of a pro-nilpotent Lie (respectively, Leibniz) algebras are finitedimensional and nilpotent. In particular, any pro-nilpotent Lie (respectively, Leibniz) algebra is finitely generated.

Due to the inclusions $L^{[i]} \subseteq L^{2^{i-1}}$ one has $\bigcap_{i=1}^{\infty} L^{[i]} \subseteq \bigcap_{i=1}^{\infty} L^{2^{i-1}} \subseteq \bigcap_{i=1}^{\infty} L^{i}=0$. Therefore, a residually nilpotent algebra is residually solvable.

Definition 2.10. A linear map $\rho: L \rightarrow L$ is called residually nilpotent, if $\bigcap_{i=1}^{\infty}$ Im $\rho^{i}=0$ holds true.

Below we introduce the analogue of the notion of nil-independency that has played a crucial role in the description of finite-dimensional solvable Lie algebras (see [25]).

Definition 2.11. Derivations $d_{1}, d_{2}, \ldots, d_{n}$ of Leibniz algebra $L$ over a field $\mathbb{F}$ are said to be residually nil-independent, if a map $f=\alpha_{1} d_{1}+\alpha_{2} d_{2}+\ldots+\alpha_{n} d_{n}$ is not residually nilpotent for any scalars $\alpha_{1}, \alpha_{2}, \ldots, \alpha_{n} \in \mathbb{F}$. In other words, $\bigcap_{i=1}^{\infty}$ Im $f^{i}=0$ if and only if $\alpha_{1}=\alpha_{2}=\cdots=\alpha_{n}=0$.

Just to recall that low-order Chevalley cohomology groups of Lie algebras is interpreted as follows

$$
\mathrm{H}^{1}(L, L)=\operatorname{Der}(L) / \operatorname{Inder}(L) \quad \text { and } \quad \mathrm{H}^{2}(L, L)=\mathrm{Z}^{2}(L, L) / \mathrm{B}^{2}(L, L)
$$

where the set $\mathrm{Z}^{2}(L, L)$ consists of those elements $\varphi \in \operatorname{Hom}\left(\wedge^{2} L, L\right)$ such that

$$
Z(x, y, z)=[x, \varphi(y, z)]-[\varphi(x, y), z]+[\varphi(x, z), y]+\varphi(x,[y, z])-\varphi([x, y], z)+\varphi([x, z], y)=0,
$$

while $\mathrm{B}^{2}(L, L)$ is the set of those elements $\psi \in \operatorname{Hom}\left(\wedge^{2} L, L\right)$ there exixts $f \in \operatorname{Hom}(L, L)$ such that

$$
\psi(x, y)=f([x, y])-[f(x), y]-[x, f(y)] .
$$

An analogue of the above for Leibniz algebras is interpreted similarly. The difference is just to replace $\mathrm{Z}^{2}(L, L)$ by $\mathrm{ZL}^{2}(L, L)$ consisting of elements $\psi \in \operatorname{Hom}(L \otimes L, L)$ such that

$$
Z(x, y, z)=[x, \varphi(y, z)]-[\varphi(x, y), z]+[\varphi(x, z), y]+\varphi(x,[y, z])-\varphi([x, y], z)+\varphi([x, z], y)=0,
$$

and $\mathrm{B}^{2}(L, L)$ must be replaced by $\operatorname{BL}^{2}(L, L)$ consisting of $\varphi \in \operatorname{Hom}(L \otimes L, L)$ satisfying the same condition (2.3) above.

$$
\operatorname{HL}^{1}(L, L)=\operatorname{Der}(L) / \operatorname{Inder}(L) \quad \text { and } \quad \operatorname{HL}^{2}(L, L)=\mathrm{ZL}^{2}(L, L) / \mathrm{BL}^{2}(L, L) .
$$

In terms of the cohomology groups the notion of completeness of a Lie (respectively, Leibniz) algebra $L$ means that it is centerless and $\mathrm{H}^{1}(L, L)=0$ (respectively, $\operatorname{HL}^{1}(L, L)=0$ ). The rigidity of a Lie (respectively, Leibniz) algebra stands for $\mathrm{H}^{2}(L, L)=0$ (respectively, $\operatorname{HL}^{2}(L, L)=0$ ).

It was observed that there is only one, so far, complete and rigid solvable Leibniz algebra with the property that the co-dimension of the nilradical is less than the rank. Such an algebra has been given in 21] by the following table of multiplications:

$$
L: \begin{cases}{\left[e_{1}, e_{1}\right]=e_{3},} & {\left[e_{i}, e_{1}\right]=e_{i+1}, \quad 2 \leq i \leq n-1,} \\ {\left[e_{1}, x\right]=-e_{1},} & {\left[e_{2}, x\right]=-e_{2}+e_{n},} \\ {\left[x, e_{1}\right]=e_{1},} & {\left[e_{i}, x\right]=-(i-1) e_{i}, \quad 3 \leq i \leq n .}\end{cases}
$$

We make use results of [3], where authors considered the algebra $\mathfrak{m}_{0}$ with the following table of multiplication on a basis $\left\{e_{1}, e_{2}, \ldots, e_{n}\right\}$

$$
\mathfrak{m}_{0}:\left\{\left[e_{i}, e_{1}\right]=e_{i+1}, \quad i \geq 2\right.
$$


and the following results have been obtained.

Proposition 2.12. The space of derivations of the algebra $\mathfrak{m}_{0}$ is the following:

$$
\operatorname{Der}\left(\mathfrak{m}_{0}\right):\left\{\begin{aligned}
d\left(e_{1}\right) & =\sum_{i=1}^{t} \alpha_{i} e_{i}, \\
d\left(e_{k}\right) & =\left((k-2) \alpha_{1}+\beta_{2}\right) e_{k}+\sum_{i=3}^{t} \beta_{i} e_{i+k-2}, \quad \text { where } k \geq 2 .
\end{aligned}\right.
$$

Note that $t \in \mathbb{N}$ in Proposition 2.12 stands for the maximal index with non-zero coefficient in the expansion by the basis, i.e., $\alpha_{i}=0$ for all $i>t$. The same is applied throughout the paper when the upper limit of a summation is given as a variable.

Lemma 2.13. Let $M$ be a residually solvable Lie algebra whose maximal by inclusion pro-nilpotent ideal is $\mathfrak{m}_{0}$ and $Q$ be the subspace complementary to $\mathfrak{m}_{0}$. Then one has $\bigcap_{i=1}^{\infty}$ Im ad $d_{x}^{i} \neq 0$ for every $x \in Q$.

Theorem 2.14. Let $M$ be an residually solvable Lie algebra whose maximal by inclusion pro-nilpotent ideal is $\mathfrak{m}_{0}$ and $Q$ be the subspace complementary to $\mathfrak{m}_{0}$. Then the dimension of $Q$ is not greater than the maximal number of residually nil-independent derivations of $\mathfrak{m}_{0}$.

Corollary 2.15. The maximal number of residually nil-independent derivations of $\mathfrak{m}_{0}$ equals two.

Therefore, due to Corollary 2.15 the dimension of $Q$ is not greater than 2 . The case $\operatorname{dim} Q=2$ has been treated in [3].

\section{Main Results}

3.1. Infinite-dimensional Lie algebras. The section we begin with treating the case $\operatorname{dim} Q=1$ of the discussion at the end of the previous section, i.e., $M=\mathfrak{m}_{0} \oplus Q$, where $\operatorname{dim} Q=1$. Further, throughout the paper a residually solvable algebra whose maximal by inclusion pro-nilpotent ideal is $N$ and the codimension of $N$ is $n$ is denoted by $R(N, n)$.

Theorem 3.1. Let $R\left(\mathfrak{m}_{0}, 1\right)$ be a residually solvable Lie algebra whose maximal by inclusion pronilpotent ideal is $\mathfrak{m}_{0}$. Then it admits a basis $\left\{x, e_{1}, e_{2}, \ldots\right\}$ such that the table of multiplications of $R\left(\mathfrak{m}_{0}, 1\right)$ on this basis is given by one of the following form

$$
R_{1}\left(\mathfrak{m}_{0}, 1, \beta\right): \begin{cases}{\left[e_{i}, e_{1}\right]=e_{i+1},} & i \geq 2, \\ {\left[e_{1}, x\right]=e_{1},} & \\ {\left[e_{i}, x\right]=(i-2) e_{i}+\sum_{k=3}^{t} \beta_{k} e_{i+k-2},} & i \geq 2,\end{cases}
$$

where $\beta=\left(\beta_{3}, \beta_{4}, \ldots, \beta_{t}\right) \in \mathbb{C}^{t-2}$ for some $t \in \mathbb{N}$.

$$
R_{2}\left(\mathfrak{m}_{0}, 1, \beta\right): \begin{cases}{\left[e_{i}, e_{1}\right]=e_{i+1},} & i \geq 2, \\ {\left[e_{1}, x\right]=e_{1}+\alpha_{2} e_{2},} & \\ {\left[e_{i}, x\right]=\left((i-2)+\beta_{2}\right) e_{i}+\sum_{k=3}^{t} \beta_{k} e_{i+k-2},} & i \geq 2,\end{cases}
$$

where $\beta=\left(\beta_{2}, \beta_{3}, \ldots, \beta_{t}\right) \in \mathbb{C}^{t-1}$ for some $t \in \mathbb{N}$.

$$
R_{3}\left(\mathfrak{m}_{0}, 1, \beta\right): \begin{cases}{\left[e_{i}, e_{1}\right]=e_{i+1},} & i \geq 2, \\ {\left[e_{i}, x\right]=e_{i}+\sum_{k=3}^{t} \beta_{k} e_{i+k-2},} & i \geq 2,\end{cases}
$$

where $\beta=\left(\beta_{3}, \beta_{4}, \ldots, \beta_{t}\right) \in \mathbb{C}^{t-2}$ for some $t \in \mathbb{N}$.

Proof. Remind that $R\left(\mathfrak{m}_{0}, 1\right)=\mathfrak{m}_{\mathfrak{o}} \oplus Q$ is the solvable Lie algebra, where $\mathfrak{m}_{0}$ is the nilradical of $R\left(\mathfrak{m}_{0}, 1\right)$ and $Q$ is the complementary subspace to $\mathfrak{m}_{0}$. Note that for any $x \in Q$ the inner derivation $\left.a d_{x}\right|_{\mathfrak{m}_{0}}$ is non-nilpotent, therefore we can write the table of multiplications of $R\left(\mathfrak{m}_{0}, 1\right)$ as follows

$$
\left[e_{1}, x\right]=\sum_{k=1}^{t} \alpha_{k} e_{k}, \quad\left[e_{i}, x\right]=\left((i-2) \alpha_{1}+\beta_{2}\right) e_{i}+\sum_{k=3}^{t} \beta_{k} e_{i+k-2}, \quad \text { where } k \geq 2 .
$$

Since $\mathfrak{m}_{0}$ is maximal by inclusion pro-nilpotent ideal, the algebras in Theorem 3.1 are obtained via case by case consideration with respect to the parameters $\alpha_{1}$ and $\beta_{2}$ as follows. 
Case 1. Let $\alpha_{1} \neq 0$. Then scaling $x$ by $\frac{1}{\alpha_{1}}$ we get

$$
\left[e_{1}, x\right]=e_{1}+\sum_{k=2}^{t} \alpha_{k} e_{k}, \quad\left[e_{i}, x\right]=\left((i-2)+\beta_{2}\right) e_{i}+\sum_{k=3}^{t} \beta_{k} e_{i+k-2}, \quad \text { where } k \geq 2 .
$$

Case 1.1. Let $\beta_{2}=0$. Setting $e_{1}^{\prime}=e_{1}+\alpha_{2} e_{2}$, without loss of generality, we can write

$$
\left[e_{1}, x\right]=e_{1}+\sum_{k=3}^{t} \alpha_{k} e_{k}, \quad\left[e_{i}, x\right]=(i-2) e_{i}+\sum_{k=3}^{t} \beta_{k} e_{i+k-2}, i \geq 2 .
$$

Then the base change $x^{\prime}=x+\sum_{k=2}^{t} \alpha_{k+1} e_{k}$ (the other basis vectors being unchanged) leads us to the required table of multiplications of $R_{1}\left(\mathfrak{m}_{0}, 1, \beta\right)$. Note that the same is applied through the paper if only one basis vector's change is given.

Case 1.2. Let $\beta_{2} \neq 0$. Then by changing $x$ as $x^{\prime}=x+\sum_{k=2}^{t-1} \alpha_{k+1} e_{k}$, we get the required table of multiplications of $R_{2}\left(\mathfrak{m}_{0}, 1, \beta\right)$.

Case 2. Let now $\alpha_{1}=0$. Then $\beta_{2} \neq 0$ (the otherwise case contradicts the maximal pro-nilpotency of $\left.\mathfrak{m}_{0}\right)$. If we scale $x$ by $\frac{1}{\beta_{2}}$ then the relations are easily computed to be

$$
\left[e_{1}, x\right]=\sum_{k=2}^{t} \alpha_{k} e_{k}, \quad\left[e_{i}, x\right]=e_{i}+\sum_{k=3}^{t} \beta_{k} e_{i+k-2}, \quad i \geq 2 .
$$

Let set $e_{1}^{\prime}=e_{1}-\alpha_{2} e_{2}$. Then without loss of generality we write $\left[e_{1}, x\right]=\sum_{k=3}^{t+1} \alpha_{k} e_{k}$ and applying the base change $x^{\prime}=x+\sum_{k=2}^{t} \alpha_{k+1} e_{k}$ we get the table of multiplication as in $R_{3}\left(\mathfrak{m}_{0}, 1, \beta\right)$.

The following proposition shows that the algebras $R_{1}\left(\mathfrak{m}_{0}, 1, \beta\right), R_{2}\left(\mathfrak{m}_{0}, 1, \beta\right)$ and $R_{3}\left(\mathfrak{m}_{0}, 1, \beta\right)$ are not complete.

\section{Proposition 3.2.}

- $d\left(e_{i}\right)=e_{i}, i \geq 2$ is an outer derivation of the algebra $R_{1}\left(\mathfrak{m}_{0}, 1, \beta\right)$;

- The derivations

$$
\begin{aligned}
& *\left\{\begin{aligned}
d\left(e_{1}\right) & =e_{2}, \\
d(x) & =\sum_{i=2}^{s-1} \beta_{i+1} e_{i},
\end{aligned}\right. \\
& *\left\{\begin{array}{l}
d\left(e_{1}\right)=\frac{\alpha_{2}}{\beta_{2}-1} e_{2}, \\
d\left(e_{i}\right)=e_{i}, i \geq 2, \\
d(x)=\sum_{i=2}^{s-1} \frac{\alpha_{2} \beta_{i+1}}{\beta_{2}-1} e_{i},
\end{array}\right.
\end{aligned}
$$

are outer derivations of the algebra $R_{2}\left(\mathfrak{m}_{0}, 1, \beta\right)$, respectively

- $d\left(e_{i}\right)=e_{i+2}, i \geq 2$, is an outer derivation of the algebra $R_{3}\left(\mathfrak{m}_{0}, 1, \beta\right)$.

Proof. It is obvious that $d\left(e_{i}\right)=e_{i}, i \geq 2$ is a derivation of $R_{1}\left(\mathfrak{m}_{0}, 1, \beta\right)$. Moreover, for any $a \in$ $R_{1}\left(\mathfrak{m}_{0}, 1, \beta\right)$ we have $a d_{a}\left(R_{1}\left(\mathfrak{m}_{0}, 1, \beta\right)\right) \subseteq\left[R_{1}\left(\mathfrak{m}_{0}, 1, \beta\right), R_{1}\left(\mathfrak{m}_{0}, 1, \beta\right)\right]=R_{1}\left(\mathfrak{m}_{0}, 1, \beta\right) \backslash\left\{e_{2}\right\}$. Thus, $d$ is not inner derivation of $R_{1}\left(\mathfrak{m}_{0}, 1, \beta\right)$.

The similar argument can be applied to prove the rest parts of the proposition.

Recall that in [3], the following residually solvable Lie algebra $R\left(\mathfrak{m}_{0}, 2, \beta\right)$, with a property that the codimension of $\mathfrak{m}_{0}$ is maximal, was obtained

$$
R\left(\mathfrak{m}_{0}, 2, \beta\right):\left\{\begin{array}{l}
{\left[e_{i}, e_{1}\right]=e_{i+1}, \quad i \geq 2} \\
{\left[e_{1}, x\right]=e_{1},} \\
{\left[e_{i}, x\right]=(i-1) e_{i}+\sum_{q=3}^{t} \beta_{q} e_{q+i-2}, \quad i \geq 2} \\
{\left[e_{i}, y\right]=e_{i}, \quad i \geq 2,}
\end{array}\right.
$$


where $\beta=\left(\beta_{3}, \beta_{4}, \ldots, \beta_{t}\right) \in \mathbb{C}^{t-2}$ for some $t \in \mathbb{N}$, where $\{x, y\}$ is the basis of $Q$ and $\left\{e_{1}, e_{2}, \ldots\right\}$ is basis of $\mathfrak{m}_{0}$. Here is a theorem on the derivations of $R\left(\mathfrak{m}_{0}, 2, \beta\right)$.

Theorem 3.3. An arbitrary algebra of the family $R\left(\mathfrak{m}_{0}, 2, \beta\right)$ is complete.

Proof. The fact that the center is trivial can be easily obtained by using the table multiplications. We prove that all derivations of $R\left(\mathfrak{m}_{0}, 2, \beta\right)$ are inner. Note that $\left\{e_{1}, e_{2}, x, y\right\}$ are generators of $R\left(\mathfrak{m}_{0}, 2, \beta\right)$. Since a derivation is completely determined by its values on generators it is sufficient to prove the existence of $a \in R\left(\mathfrak{m}_{0}, 2, \beta\right)$ such that $d(z)=a d_{a}(z)$, where $z$ is either of $e_{1}, e_{2}, x, y$.

Observe that for any $k \in \mathbb{N}$ the quotient algebra

$$
\left(R\left(\mathfrak{m}_{0}, 2, \beta\right)\right)_{k}:=R\left(\mathfrak{m}_{0}, 2, \beta\right) / \mathfrak{m}_{0}^{k}=\mathfrak{m}_{0} / \mathfrak{m}_{0}^{k} \oplus Q=\overline{\mathfrak{m}}_{0} \oplus Q \text { with } \mathfrak{m}_{0}^{k}=\operatorname{Span}\left\{e_{k+1}, \ldots\right\}
$$

is finite-dimensional solvable Lie algebra, which is maximal solvable extension of the nilpotent Lie algebra $\mathfrak{m}_{0}$. Since such an algebra is unique up to isomorphism the algebra $\left(R\left(\mathfrak{m}_{0}, 2, \beta\right)\right)_{k}$ must be isomorphic to $\left(R\left(\mathfrak{m}_{0}, 2,0\right)\right)_{k}$. However, according to 4, all its derivations are inner.

Let $d \in \operatorname{Der}\left(R\left(\mathfrak{m}_{0}, 2, \beta\right)\right)$ and introduce $\bar{d} \in \operatorname{Der}\left(\left(R\left(\mathfrak{m}_{0}, 2, \beta\right)\right)_{k}\right)$ such that $\bar{d}(\bar{v})=\overline{d(v)}, \quad \bar{v}=v+\mathfrak{m}_{0}^{k}$. The function $\bar{d}(\bar{v})$ is well-defined, i.e., $\mathfrak{m}_{0}^{k}$ is invariant under $d$.

Indeed,

$$
d\left(\mathfrak{m}_{0}\right)=d_{\mathfrak{m}_{0}}\left(\mathfrak{m}_{0}\right)+d_{Q}\left(\mathfrak{m}_{0}\right), \text { where } d_{\mathfrak{m}_{0}}: \mathfrak{m}_{0} \rightarrow \mathfrak{m}_{0}, \quad d_{Q}: \mathfrak{m}_{0} \rightarrow Q
$$

Taking into account that $[Q, Q]=0$ and $\mathfrak{m}_{0}$ is an ideal of $R\left(\mathfrak{m}_{0}, 2, \beta\right)$ such that $\left[\mathfrak{m}_{0}, Q\right]=\mathfrak{m}_{0}$ we derive

$$
d\left(\mathfrak{m}_{0}\right)=d\left(\left[\mathfrak{m}_{0}, Q\right]\right)=\left[d\left(\mathfrak{m}_{0}\right), Q\right]+\left[\mathfrak{m}_{0}, d(Q)\right]=\left[d_{\mathfrak{m}_{0}}\left(\mathfrak{m}_{0}\right), Q\right]+\left[\mathfrak{m}_{0}, d(Q)\right] \subseteq \mathfrak{m}_{0}
$$

Consequently, we get

$$
d_{Q}\left(\mathfrak{m}_{0}\right)=0, \quad d\left(\mathfrak{m}_{0}\right) \subseteq \mathfrak{m}_{0}
$$

These imply $d\left(\mathfrak{m}_{0}^{k}\right) \subseteq \mathfrak{m}_{0}^{k}$ for any $k \in \mathbb{N}$. Thus the well-definedness of $\bar{d}$ is shown.

Set

$d\left(e_{1}\right)=\sum_{i=1}^{s} a_{i} e_{i}, \quad d\left(e_{2}\right)=\sum_{i=1}^{s} b_{i} e_{i}, \quad d(x)=\sum_{i=1}^{s} \gamma_{i} e_{i}+\gamma_{1,1} x+\gamma_{2,2} y, \quad d(y)=\sum_{i=1}^{s} \tau_{i} e_{i}+\tau_{1,1} x+\tau_{2,2} y$.

Let us take $k \geq \max \{s, t\}$. Then $\overline{d(v)}=\bar{d}(\bar{v})=a d_{\bar{c}_{k}}$ for some $\bar{c}_{k}=c_{k}+\mathfrak{m}_{0}^{k}$ and for any $\bar{v}=v+\mathfrak{m}_{0}^{k}$.

Let

$$
\bar{c}_{k}=\sum_{i=1}^{k} \alpha_{k, i} e_{i}+\lambda_{k} x+\mu_{k} y+\mathfrak{m}_{0}^{k} .
$$

Then from the equalities

$$
\overline{d\left(e_{1}\right)}=\left[\bar{e}_{1}, \bar{c}_{k}\right], \quad \overline{d\left(e_{2}\right)}=\left[\bar{e}_{2}, \bar{c}_{k}\right], \quad \overline{d(y)}=\left[\bar{y}, \bar{c}_{k}\right]
$$

we get

$$
\begin{aligned}
& \sum_{i=1}^{s} a_{i} e_{i}-\lambda_{k} e_{1}+\sum_{i=2}^{k-1} \alpha_{k, i} e_{i+1} \in \mathfrak{m}_{0}^{k}, \\
& \sum_{i=1}^{s} b_{i} e_{i}-\left(\lambda_{k}+\mu_{k}\right) e_{2}-\left(a_{k, 1}+\beta_{3}\right) e_{3}-\lambda_{k} \sum_{q=4}^{t} \beta_{q} e_{q} \in \mathfrak{m}_{0}^{k}, \\
& \sum_{i=1}^{s} \tau_{i} e_{i}+\tau_{1,1} x+\tau_{2,2} y+\sum_{i=2}^{k} \alpha_{k, i} e_{i} \in \mathfrak{m}_{0}^{k} .
\end{aligned}
$$

Comparing the coefficients of the basis vectors we derive

$$
\left\{\begin{array}{l}
a_{1}=\lambda_{k}, \quad a_{i}=-\alpha_{k, i-1}, \quad 3 \leq i \leq s, \quad \alpha_{k, i}=0, \quad s \leq j \leq k-1, \\
b_{2}=\lambda_{k}+\mu_{k}, \quad b_{3}=\alpha_{k, 1}+\beta_{3} . \\
\tau_{i}=-\alpha_{k, i}, \quad 2 \leq i \leq s-1, \quad \alpha_{k, k}=0
\end{array}\right.
$$

From (3.1) we obtain $c_{k}=\left(b_{3}-\beta_{3}\right) e_{1}-\sum_{i=2}^{s-1} a_{i+1} e_{i}+a_{1} x+\left(b_{2}-a_{1}\right) y$. Hence, $c_{k}$ depends only on the parameters $a_{i}, \tau_{i}, b_{1}, b_{2}, 1 \leq i \leq s$. Therefore, $c_{k}=c_{k+1}$ for any $k \geq \max \{s, t\}$. Thus, setting $c:=c_{k}$ and $W_{k}=\operatorname{Span}\left\{x, y, e_{1}, \ldots, e_{k}\right\}$ we get $d(z)_{\mid W_{k}}=a d_{c}(z)_{\mid W_{k}}$ for $z \in\left\{e_{1}, e_{2}, x, y\right\}$ and $k \geq \max \{s, t\}$. Now taking into account that $\bigcup_{k=1}^{\infty} W_{k}=R\left(\mathfrak{m}_{0}, 2, \beta\right)$ we obtain $d=a d_{c}$.

Let us now treat the second cohomology groups of $R\left(\mathfrak{m}_{0}, 2, \beta\right)$.

Theorem 3.4. The second cohomology groups $H^{2}\left(R\left(\mathfrak{m}_{0}, 2, \beta\right), R\left(\mathfrak{m}_{0}, 2, \beta\right)\right)$ of $R\left(\mathfrak{m}_{0}, 2, \beta\right)$ are trivial. 
Proof. Let $\varphi \in Z^{2}\left(R\left(\mathfrak{m}_{0}, 2, \beta\right), R\left(\mathfrak{m}_{0}, 2, \beta\right)\right)$. We prove that there exists a $f \in \operatorname{Hom}\left(R\left(\mathfrak{m}_{0}, 2, \beta\right), R\left(\mathfrak{m}_{0}, 2, \beta\right)\right)$ that generates $\varphi$ as a coboundry. An element $\varphi$ of $Z^{2}\left(R\left(\mathfrak{m}_{0}, 2, \beta\right), R\left(\mathfrak{m}_{0}, 2, \beta\right)\right)$ on the basis $\left\{x, y, e_{1}, e_{2}, \ldots\right\}$ is written in the form

$$
\begin{array}{ll}
\varphi\left(e_{i}, e_{j}\right)=\sum_{k=1}^{p_{i, j}} a_{k}^{i, j} e_{k}+a_{1,1}^{i, j} x+a_{2,2}^{i, j} y, & \varphi\left(e_{i}, x\right)=\sum_{k=1}^{p_{i}} b_{k}^{i} e_{k}+b_{1,1}^{i} x+b_{2,2}^{i} y, \\
\varphi\left(e_{i}, y\right)=\sum_{k=1}^{q_{i}} c_{k}^{i} e_{k}+c_{1,1}^{i} x+c_{2,2}^{i} y, & \varphi(x, y)=\sum_{k=1}^{s} g_{k} e_{k}+g_{1,1} x+g_{2,2} y, \quad i \geq 1 .
\end{array}
$$

We choose $f \in \operatorname{Hom}\left(R\left(\mathfrak{m}_{0}, 2, \beta\right), R\left(\mathfrak{m}_{0}, 2, \beta\right)\right)$ as follows

$$
\begin{aligned}
& f\left(e_{1}\right)=\tau_{1}^{1} e_{1}-c_{2}^{1} e_{2}-\sum_{k=3}^{s_{1}} \tau_{k}^{1} e_{k}+b_{1,1}^{1} x+b_{2,2}^{1} y \\
& f\left(e_{i}\right)=c_{1}^{i} e_{1}+\sum_{k=2}^{s_{i}} \tau_{k}^{i} e_{k}+c_{1,1}^{i} x+c_{2,2}^{i} y \\
& f(x)=\mu_{1} e_{1}+\sum_{k=2}^{s_{1}-1}\left(b_{k+1}^{1}-c_{2}^{1} \beta_{k+1}+(k-1) \tau_{k+1}^{1}+\sum_{i=3}^{k} \tau_{i}^{1} \beta_{k+3-i}\right) e_{k}-b_{1}^{1} x+\mu_{2,2} y, \\
& f(y)=g_{1} e_{1}+\sum_{k=2}^{s}\left(c_{k+1}^{1}+\tau_{k+1}^{1}\right) e_{k}-c_{1}^{1} x+\nu_{2,2} y, s=\max \left\{q_{1}, s_{1}\right\} .
\end{aligned}
$$

Consider the cocycle $\chi=\varphi-\psi \in Z^{2}\left(R\left(\mathfrak{m}_{0}, 2, \beta\right), R\left(\mathfrak{m}_{0}, 2, \beta\right)\right)$, where $\psi(x, y)=f([x, y])-[f(x), y]-$ $[x, f(y)]$ and show that $\chi$ is trivial. In the expension of the cocycle $\chi$ via the basis its components will be written as functions of the components of $f$ and $\varphi$

$$
\begin{aligned}
& \chi\left(e_{1}, x\right)=b_{2}^{1} e_{2}, \quad \chi\left(e_{1}, y\right)=c_{1,1}^{1} x+c_{2,2}^{1} y, \\
& \chi(x, y)=\sum_{k=2}^{s} g_{k}^{\prime} e_{k}+g_{1,1}^{\prime} x+g_{2,2}^{\prime} y, \\
& \chi\left(e_{i}, e_{j}\right)=\sum_{k=1}^{p_{i, j}} a_{k}^{i, j} e_{k}+a_{1,1}^{i, j} x+a_{2,2}^{i, j} y, \quad i \geq 1, \\
& \chi\left(e_{i}, x\right)=\sum_{k=1}^{p_{i}} b_{k}^{i} e_{k}+b_{1,1}^{i} x+b_{2,2}^{i} y, \quad i \geq 2, \\
& \chi\left(e_{i}, y\right)=\sum_{k=2}^{q_{i}} c_{k}^{i} e_{k}+g_{1} e_{i+1}-c_{1}^{1}\left((i-2) e_{i}+\sum_{k=3}^{t} \beta_{k} e_{k+i-2}\right)+\nu_{2,2} e_{i}, \quad i \geq 2 .
\end{aligned}
$$

Now we impose to $\chi$ the cocycle identities $Z=0$ (see (2.2) ) to derive the following set of constraints:

\section{2-cocyle identity}

\section{Constraints}

\begin{tabular}{ll}
\hline$Z\left(e_{1}, x, y\right)=0$, & $\Rightarrow\left\{\begin{array}{l}g_{k}^{\prime}=0,2 \leq k \leq s, g_{1,1}^{\prime}=b_{2}^{1}=c_{1,1}^{1}=c_{2,2}^{1}=0, \\
a_{k}^{i, j}=a_{1,1}^{i, j}=a_{2,2}^{i, j}=0, i, j \geq 2, k \geq 1,\end{array}\right.$ \\
$Z\left(e_{i}, e_{j}, y\right)=0, i, j \geq 2$, & $\Rightarrow\left\{\begin{array}{l}a_{1}^{i, 1}=a_{1,1}^{i, 1}=a_{2,2}^{i, 1}=0, i \geq 2, \\
c_{k}^{k}=c_{2}^{2}+(k-2) c_{1}^{1}, k \geq 3, \\
c_{k}^{i}=0, i \geq 3,2 \leq k \leq i-1, \\
c_{k}^{i}=c_{k-i+2}^{2}, k>i \geq 3,\end{array}\right.$ \\
$Z\left(e_{i}, e_{1}, y\right)=0, i \geq 2$, & $\Rightarrow\left\{\begin{array}{l}g_{2,2}=b_{1}^{i}=b_{1,1}^{i}=b_{2,2}^{i}=0, i \geq 2 .\end{array}\right.$
\end{tabular}

We let

$$
\begin{aligned}
& \tau_{i+1}^{i+1}=\tau_{i}^{i}+\tau_{1}^{1}+a_{i+1}^{i, 1}+b_{1,1} \beta_{3}, \\
& \tau_{i}^{i+1}=\tau_{i-1}^{i}+a_{i}^{i, 1}+(i-1) b_{1,1}+b_{2,2}, \quad \tau_{2}^{i+1}=a_{2}^{i, 1}, \\
& \tau_{k}^{i+1}=\tau_{k-1}^{i}+a_{k}^{i, 1}+b_{1,1} \beta_{k+2-i}, \quad 3 \leq k \leq t+i-2, \quad k \neq\{i, i+1\}, \quad i \geq 2,
\end{aligned}
$$

to get $(\varphi-\psi)\left(e_{i}, e_{1}\right)=\chi\left(e_{i}, e_{1}\right)=0, i \geq 1$. 
Letting

$$
\begin{aligned}
& \mu_{2,2}=b_{1}^{1}-b_{2}^{2}+\sum_{j=3}^{t} \beta_{j} \tau_{2}^{j}, \\
& \tau_{3}^{2}=b_{1}^{1} \beta_{3}-\mu_{1}-b_{3}^{2}-\tau_{2}^{2} \beta_{3}+\sum_{j=3}^{t} \beta_{j} \tau_{3}^{j}, \\
& \tau_{i}^{2}=\frac{1}{i-2}\left(b_{1}^{1} \beta_{i}-b_{i}^{2}-\tau_{2}^{2} \beta_{i}-\tau_{3}^{2} \beta_{i-1}-\cdots-\tau_{i-1}^{2} \beta_{3}+\sum_{j=3}^{t} \beta_{j} \tau_{i}^{j}\right), \quad i \geq 4 .
\end{aligned}
$$

we obtain $(\varphi-\psi)\left(e_{2}, x\right)=\chi\left(e_{2}, x\right)=0$.

Finally, $Z\left(e_{i}, e_{1}, x\right)=0, i \geq 2$ gives $(\varphi-\psi)\left(e_{i}, x\right)=\chi\left(e_{i}, x\right)=0, \quad i \geq 3$. This completes the proof.

3.2. Infinite-dimensional Leibniz algebra. In this section we study the extensions of a non-Lie Leibniz algebra with the following table of multiplications. (This algebra has been introduced in [31]).

$$
F:\left\{\left[e_{i}, e_{1}\right]=e_{i+1}, \quad i \geq 2 .\right.
$$

The algebra of derivations of $F$ is given by the following proposition, the proof of that is immediate if we apply the derivation rules to the table of multiplications above.

Proposition 3.5. The derivations of the algerba $F$ are given as follows:

$$
\operatorname{Der}(F):\left\{\begin{array}{l}
d\left(e_{1}\right)=\alpha_{1} e_{1}, \\
d\left(e_{i}\right)=\left((i-2) \alpha_{1}+\beta_{2}\right) e_{i}+\sum_{k=3}^{t} \beta_{k} e_{k+i-2}, \quad i \geq 2,
\end{array}\right.
$$

Let $R(F)$ stand for a family of residually solvable Leibniz algebras whose maximal by inclusion pro-nilpotent ideal is $F$ and $Q$ be the subspace complementary to $F$, i.e., $R(F)=F \oplus Q$.

Lemma 3.6. The derivations $a d_{x}$ are non-residually nilpotent for any $x \in Q$.

Proof. Let us assume the contrary that $\bigcap_{k=1}^{\infty} I m a d_{x}^{k}=0$ for some $x \in Q$. Set $R(F)=F+\mathbb{C} x$. Since $a d_{x \mid F}=d$ for some $d \in \operatorname{Der}(F)$ the condition $\bigcap_{k=1}^{\infty} \operatorname{Im} a d_{x}^{k}=0$ implies $\alpha_{1}=\beta_{2}=0$ (see Proposition 3.5). Therefore, $\left[e_{1}, x\right]=0, \quad\left[e_{i}, x\right]=\sum_{k=3}^{t} \beta_{k} e_{i+k-2}, \quad i \geq 2$ and $\bigcap_{i=1}^{\infty}(R(F))^{i}=0$, i.e., $R(F)$ is pro-nilpotent which contradicts to the maximality of $F$.

The fact that the maximal number of residually nil-independent derivations of $F$ is equal to 2 implies that the dimension of the subspace $Q$ of $R(F)$ is not greater than 2 . Let consider the case $\operatorname{dim} Q=1$.

Theorem 3.7. The algebra $R(F, 1)$ admits a basis $\left\{x, e_{1}, e_{2}, \ldots\right\}$ such that the table of multiplications of $R(F, 1)$ on this basis has one of the following forms:

where $\beta=\left(\beta_{2}, \beta_{3}, \ldots, \beta_{t}\right) \in \mathbb{C}^{t-1}$ for some $t \in \mathbb{N}$.

$$
R_{1}(F, 1, \beta):\left\{\begin{array}{l}
{\left[e_{i}, e_{1}\right]=e_{i+1}, \quad i \geq 2,} \\
{\left[e_{1}, x\right]=-\left[x, e_{1}\right]=e_{1},} \\
{\left[e_{i}, x\right]=\left(i-2+\beta_{2}\right) e_{i}+\sum_{k=3}^{t} \beta_{k} e_{k+i-2}, \quad i \geq 2 .}
\end{array}\right.
$$

$$
R_{2}(F, 1, \beta):\left\{\begin{array}{l}
{\left[e_{i}, e_{1}\right]=e_{i+1}, \quad i \geq 2,} \\
{\left[x, e_{1}\right]=-e_{1}+e_{2},} \\
{\left[e_{1}, x\right]=e_{1},} \\
{\left[e_{i}, x\right]=(i-1) e_{i}+\sum_{k=3}^{t} \beta_{k} e_{k+i-2}, \quad i \geq 2,} \\
{[x, x]=\sum_{k=2}^{t-1} \beta_{k+1} e_{k} .}
\end{array}\right.
$$

where $\beta=\left(\beta_{3}, \beta_{4}, \ldots, \beta_{t}\right) \in \mathbb{C}^{t-2}$ for some $t \in \mathbb{N}$.

$$
R_{3}(F, 1, \beta):\left\{\begin{array}{l}
{\left[e_{i}, e_{1}\right]=e_{i+1}, \quad i \geq 2,} \\
{\left[e_{i}, x\right]=e_{i}+\sum_{k=3}^{t} \beta_{k} e_{k+i-2}, \quad i \geq 2,}
\end{array}\right.
$$

where $\beta=\left(\beta_{3}, \beta_{4}, \ldots, \beta_{t}\right) \in \mathbb{C}^{t-2}$ for some $t \in \mathbb{N}$. 
Proof. Since, for any $x \in Q$ the derivation $a d_{x}$ of $F$ is non-nilpotent we use it to write the products $\left[e_{1}, x\right]$ and $\left[e_{i}, x\right] \quad i \geq 2$ in $F$ as follows

$$
\left[e_{1}, x\right]=\alpha_{1} e_{1}, \quad\left[e_{i}, x\right]=\left((i-2) \alpha_{1}+\beta_{2}\right) e_{i}+\sum_{k=3}^{t} \beta_{k} e_{k+i-2}, \quad i \geq 2 .
$$

Let

$$
\left[x, e_{i}\right]=\sum_{k=1}^{t} \gamma_{i, k} e_{k}, \quad i \in\{1,2\}, \quad[x, x]=\sum_{k=1}^{t} \mu_{k} e_{k}+\mu x .
$$

Case 1. Let $\alpha_{1} \neq 0$. Taking $x^{\prime}=\frac{x}{\alpha_{1}}$ we write $\left[e_{1}, x\right]=e_{1}$. Since $\left[e_{i},[x, x]\right]=0, i \in\{1,2\}$, we get $\mu=\mu_{1}=0$. And $\operatorname{Leib}\left(x, e_{1}, e_{2}\right)=\operatorname{Leib}\left(e_{2}, x, e_{2}\right)=0$ gives $\left[x, e_{2}\right]=0$.

Note that the base change $x^{\prime}=x-\sum_{k=2}^{t-1} \gamma_{1, k+1} e_{k}$ gives $\left[x^{\prime}, e_{1}\right]=\gamma_{1,1} e_{1}+\gamma_{1,2} e_{2}$. Since $e_{1} \notin$ $A n n_{r}(R(F, 1))$ and $e_{i} \in A n n_{r}(R(F, 1)), i \geq 3$ we obtain $\gamma_{1,1}=-1$ and $\left[x, e_{i}\right]=0$ for $i \geq 3$.

By using $\operatorname{Leib}\left(x, x, e_{1}\right)=0$, we get

$$
\gamma_{1,2}\left(\beta_{2}-1\right)=0, \quad \mu_{k}=\gamma_{1,2} \beta_{k+1}, \quad 2 \leq k \leq t-1, \quad \mu_{t}=0 .
$$

Case 1.1. If $\gamma_{1,2}=0$ then $\mu_{k}=0,2 \leq k \leq t$ and this leads to $R_{1}(F, 1, \beta)$.

Case 1.2. If $\gamma_{1,2} \neq 0$ then $\beta_{2}=1$. The base change $e_{1}^{\prime}=e_{1}$ and $e_{i}^{\prime}=\gamma_{1,2} e_{i}, \quad i \geq 2$ leads to the table of multiplications of $R_{2}(F, 1, \beta)$.

Case 2. Let now $\alpha_{1}=0$. Then $\beta_{2} \neq 0$ (otherwise, $F$ would be non-maximal pro-nilpotent ideal ). Let $x^{\prime}=\frac{x}{\beta_{2}}$ to get

$$
\left[e_{i}, x\right]=e_{i}+\sum_{k=3}^{t} \beta_{k} e_{k+i-2}, \quad i \geq 2, \quad\left[x, e_{i}\right]=\sum_{k=1}^{t} \gamma_{i, k} e_{k}, \quad i \in\{1,2\}, \quad[x, x]=\sum_{k=1}^{t} \mu_{k} e_{k}+\mu x
$$

Since $\left[e_{2},[x, x]\right]=0$ we obtain $\mu=\mu_{1}=0$, hence, $[x, x]=\sum_{k=2}^{t} \mu_{k} e_{k}$.

The base change $x^{\prime}=x-\sum_{k=2}^{t-1} \gamma_{1, k+1} e_{k}$, gives $\left[x^{\prime}, e_{1}\right]=\gamma_{1,1} e_{1}+\gamma_{1,2} e_{2}$. Moreover, Leib $\left(e_{2}, x, e_{1}\right)=$ $\operatorname{Leib}\left(x, e_{1}, e_{2}\right)=0$ implies $\gamma_{1,1}=\gamma_{2, k}=0,2 \leq k \leq t$. Hence, $\left[x, e_{2}\right]=\gamma_{2,1} e_{1}$.

Since $[a, b]+[b, a] \in A n n_{r}(R(F, 1))$ for any $a, b \in R(F, 1)$, we get $e_{i} \in R(F, 1), i \geq 3$. This means that $\left[x, e_{i}\right]=0, i \geq 3$. Therefore, from

$$
\left[x,\left[e_{1}, x\right]\right]-\left[\left[x, e_{1}\right], x\right]+\left[[x, x], e_{1}\right]=0
$$

we get

$$
0-\gamma_{1,2}\left(e_{2}+\sum_{k=3}^{t} \beta_{k} e_{k}\right)+\sum_{k=2}^{t} \mu_{k} e_{k+1}=0
$$

hence

$$
\gamma_{1,2}=0, \mu_{k}=0,2 \leq k \leq t \text { and thus }[x, x]=0 .
$$

From the identity $\operatorname{Leib}\left(x, e_{i}, x\right)=0, i \in\{1,2\}$ we obtain $[x, x]=\left[x, e_{2}\right]=0$. We are left with $\left[e_{i}, x\right]=e_{i}+\sum_{k=3}^{t} \beta_{k} e_{k+i-2}, \quad i \geq 2$ and this completes the proof.

The following proposition shows that two of the family of algebras obtained are not complete.

Proposition 3.8. The derivations

- $d\left(e_{i}\right)=e_{i}, i \geq 2$

and

- $d\left(e_{i}\right)=e_{i+2}, i \geq 2$,

are outer derivations of the algebras $R_{1}(F, 1, \beta)$ and $R_{2}(F, 1, \beta)$, respectively.

Proof. The proof of the proposition is straightforward if one uses the tables of multiplications along with the derivation rule.

Theorem 3.9. An arbitary algebra of the family $R_{2}(F, 1, \beta)$ is complete. 
Proof. The fact that the center of $R_{2}(F, 1, \beta)$ is trivial follows immediately from the table of multiplications. We prove that all derivations of $R_{2}(F, 1, \beta)$ are inner. Note that $R_{2}(F, 1, \beta)=F \oplus \mathbb{C} x$, where $Q=\mathbb{C} x$, and $\left\{e_{1}, e_{2}, x\right\}$ are generators of $R_{2}(F, 1, \beta)$. Note also that for $k \in \mathbb{N}$ the quotient algebra $R_{2}(F, 1, \beta) / F^{k}$ is isomorphic to the finite-dimensional Leibniz algebra with the table of multiplications (2.4). We make use the fact that all derivations of the algebra with the table of multiplications (2.4) are inner what was proven in 21. We set

$$
d\left(e_{1}\right)=\sum_{i=1}^{s} a_{i} e_{i}, \quad d\left(e_{2}\right)=\sum_{i=1}^{s} b_{i} e_{i}, \quad d(x)=\sum_{i=1}^{s} \gamma_{i} e_{i}+\gamma_{1,1} x+\gamma_{2,2} y
$$

and put $\quad \bar{c}_{k}=\sum_{i=1}^{k} \alpha_{k, i} e_{i}+\lambda_{k} x+F^{k}$. From the equalities $\overline{d\left(e_{1}\right)}=\left[\bar{e}_{1}, \bar{c}_{k}\right], \quad \overline{d\left(e_{2}\right)}=\left[\bar{e}_{2}, \bar{c}_{k}\right]$ and $\overline{d(x)}=\left[\bar{x}, \bar{c}_{k}\right]$ we get

$$
\sum_{i=1}^{s} a_{i} e_{i}-\lambda_{k} e_{1}, \quad \sum_{i=1}^{s} b_{i} e_{i}-\left(\lambda_{k}+\mu_{k}\right) e_{2}-\lambda_{k} \sum_{q=3}^{t} \beta_{q} e_{q}, \quad \sum_{i=1}^{s} \gamma_{i} e_{i}+\gamma_{1,1} x+\alpha_{k, 1} e_{1} \in F^{k} .
$$

This implies

$$
a_{1}=\lambda_{k}, \quad b_{2}=\lambda_{k}+\mu_{k}, \quad \gamma_{1}=-\alpha_{k, 1} .
$$

From (3.2) we conclude that $c_{k}=c_{k+1}$ for any $k \geq \max \{s, t\}$, then setting $c:=c_{k}$, for the smallest $k$ obtain $d=a d_{c}$.

By the following theorem we describe the family $R(F, 2)$ of residually solvable Leibniz algebras whose maximal by inclusion pro-nilpotent ideal is $F$ and the subspace complementary to $F$ is maximal.

Theorem 3.10. The algebra $R(F, 2)$ admits a basis $\left\{x, y, e_{1}, e_{2}, \ldots\right\}$ such that the table of multiplication of $R(F, 2)$ on this basis has the following form

$$
R(F, 2, \beta):\left\{\begin{array}{l}
{\left[e_{i}, e_{1}\right]=e_{i+1}, \quad i \geq 2,} \\
{\left[e_{1}, x\right]=-\left[x, e_{1}\right]=e_{1}} \\
{\left[e_{i}, x\right]=(i-2) e_{i}+\sum_{k=2}^{t} \beta_{k} e_{k+i-2}, \quad i \geq 2,} \\
{\left[e_{i}, y\right]=e_{i}, \quad i \geq 2,}
\end{array}\right.
$$

where $\beta=\left(\beta_{2}, \beta_{3}, \ldots, \beta_{t}\right) \in \mathbb{C}^{t-1}$ for some $t \in \mathbb{N}$.

Proof. Due to Proposition 3.5 we have

$$
\left[e_{1}, x\right]=e_{1}, \quad\left[e_{i}, x\right]=(i-2) e_{i}+\sum_{k=2}^{t} \beta_{k} e_{k+i-2}, \quad\left[e_{i}, y\right]=e_{i}+\sum_{k=2}^{t} \beta_{k}^{\prime} e_{k+i-2}, \quad i \geq 2 .
$$

Let

$$
\begin{aligned}
{\left[x, e_{i}\right] } & =\sum_{k=1}^{t} \gamma_{i, k} e_{k}, & {\left[y, e_{i}\right] } & =\sum_{k=1}^{t} \eta_{i, k} e_{k}, \quad i \in\{1,2\}, \\
{[x, y] } & =\sum_{k=1}^{t} \nu_{k} e_{k}+C_{1} x+C_{2} y, & {[y, x] } & =\sum_{k=1}^{t} \nu_{k}^{\prime} e_{k}+C_{1}^{\prime} x+C_{2}^{\prime} y, \\
{[x, x] } & =\sum_{k=1}^{t} \mu_{k} e_{k}+D_{1} x+D_{2} y, & {[y, y] } & =\sum_{k=1}^{t} \mu_{k}^{\prime} e_{k}+D_{1}^{\prime} x+D_{2}^{\prime} y .
\end{aligned}
$$

The identities $\operatorname{Leib}\left(e_{i}, Q, Q\right)=0, \quad i \in\{1,2\}$ imply $[Q, Q] \in F$. From $\operatorname{Leib}\left(e_{2}, Q, e_{i}\right)=0, i \in\{1,2\}$ we obtain $\gamma_{1,1}=-1, \gamma_{2,1}=\eta_{1,1}=\eta_{2,1}=0$. Use $\operatorname{Leib}\left(Q, e_{1}, e_{2}\right)=0$ to get $\left[Q, e_{2}\right]=0$ which is the base of induction. Hence, applying the induction we obtain $\left[Q, e_{i}\right]=0$ for $i \geq 2$.

The equalities $[x,[x, x]]=0$ and $[x,[y, y]]=0$ give $[x, x]=\sum_{k=2}^{t} \mu_{k} e_{k}$ and $[y, y]=\sum_{k=2}^{t} \mu_{k}^{\prime} e_{k}$, respectively.

The base change

$$
x^{\prime}=x-\sum_{k=2}^{t-1} \gamma_{1, k+1} e_{k}, \text { and } y^{\prime}=y-\sum_{k=2}^{t-1} \eta_{1, k+1} e_{k} .
$$

enables us to write

$$
\left[x, e_{1}\right]=-e_{1}+\gamma_{1,2} e_{2}, \quad\left[y, e_{1}\right]=\eta_{1,2} e_{2} .
$$


From the equalities $\operatorname{Leib}\left(x, e_{1}, x\right)=\operatorname{Leib}\left(y, e_{1}, y\right)=0$ we derive $\gamma_{1,2}=\eta_{1,2}=\mu_{k}=\mu_{k}^{\prime}=0,2 \leq$ $k \leq t$ which leads to $\left[x, e_{1}\right]=-e_{1}$ and $[x, x]=[y, y]=\left[y, e_{1}\right]=0$. The equalities Leib $\left(x, y, e_{1}\right)=$ $\operatorname{Leib}\left(y, x, e_{1}\right)=0$ give $[x, y]=\nu_{1} e_{1}$ and $[y, x]=\nu_{1}^{\prime} e_{1}$. Use the base change $y^{\prime}=y+\nu_{1} e_{1}$ to get $[x, y]=0, \quad[y, x]=\left(\nu_{1}^{\prime}+\nu_{1}\right) e_{1}$. The identity $\operatorname{Leib}(x, y, x)=0$ gives $[y, x]=0$.

Finally, we make use $\operatorname{Leib}\left(e_{2}, x, y\right)=0$ to get $\beta_{k}^{\prime}=0,2 \leq k \leq t$. Thus we obtain the required table of multiplications of $R(F, 2, \beta)$.

Theorem 3.11. All Leibniz algebras $R_{2}(F, 2, \beta)$ are complete .

Proof. The fact that the center trivial is obvious from the table of multiplications. Now, we prove that all derivations are inner. Recall that $R(F, 2, \beta)=F \oplus Q$, where $\{x, y\}$ is the basis of $Q$ and $\left\{e_{1}, e_{2}, x, y\right\}$ are generators of $R(F, 2, \beta)$. Consider the quotient algebra $(R(F, 2, \beta))_{k}=\bar{F} \oplus Q$, where $\bar{F}=F / F^{k}$ for $k \in \mathbb{N}$. It is isomorphic to $(R(F, 2,0))_{k}$. By using the derivation rule and the table of multiplications of $(R(F, 2,0))_{k}$ it is not hard to see that all derivation of the algebra $(R(F, 2,0))_{k}$ are inner. Therefore, all derivations of $(R(F, 2,0))_{k}=\bar{F} \oplus Q, k \in \mathbb{N}$ also are inner.

Set

$$
d\left(e_{1}\right)=\sum_{i=1}^{s} a_{i} e_{i}, \quad d\left(e_{2}\right)=\sum_{i=1}^{s} b_{i} e_{i}, \quad d(x)=\sum_{i=1}^{s} \gamma_{i} e_{i}+\gamma_{1,1} x+\gamma_{2,2} y .
$$

If we choose $\bar{c}_{k}=\sum_{i=1}^{k} \alpha_{k, i} e_{i}+\lambda_{k} x+\mu_{k} y+F^{k}$, then $\overline{d\left(e_{1}\right)}=\left[\bar{e}_{1}, \bar{c}_{k}\right], \quad \overline{d\left(e_{2}\right)}=\left[\bar{e}_{2}, \bar{c}_{k}\right], \quad \overline{d(x)}=\left[\bar{x}, \bar{c}_{k}\right]$. Hence,

$$
\sum_{i=1}^{s} a_{i} e_{i}-\lambda_{k} e_{1}, \quad \sum_{i=1}^{s} b_{i} e_{i}-\lambda_{k} \sum_{k=3}^{s} \beta_{k} e_{k}-\mu_{k} e_{2}, \quad \sum_{i=1}^{s} \gamma_{i} e_{i}+\gamma_{1,1} x+\gamma_{2,2} y+\alpha_{k, 1} e_{1} \in F^{k},
$$

This implies

$$
a_{1}=\lambda_{k}, \quad b_{2}=\mu_{k}, \quad \gamma_{1}=-\alpha_{k, 1} .
$$

Thus, $\quad c_{k}=-\gamma_{1} e_{1}+a_{1} x+b_{2} y$. From (3.3) we conclude that $c_{k}=c_{k+1}$ for any $k \geq \max \{s, t\}$. Let $l$ be the smallest $k$ satisfying this condition. Then setting $c:=c_{l}$ we get $d=a d_{c}$.

Now we treat the second cohomology groups of $R(F, 2, \beta)$.

Theorem 3.12. The second cohomology groups $H L^{2}(R(F, 2, \beta), R(F, 2, \beta))$ of the algebras $R(F, 2, \beta)$ are trivial.

Proof. We follow the same strategy as in Theorem 3.4 i.e., prove that any cocycle is generated by a linear function $f \in \operatorname{Hom}(R(F, 2, \beta), R(F, 2, \beta))$.

Let $\varphi \in Z^{2}(R(F, 2, \beta), R(F, 2, \beta))$. We expand $\varphi$ by the basis $\left\{x, y, e_{1}, e_{2}, \ldots\right\}$ as follows

$$
\begin{aligned}
& \varphi\left(e_{i}, e_{j}\right)=\sum_{k=1}^{p(i, j)} a_{k}^{i, j} e_{k}+a_{1,1}^{i, j} x+a_{2,2}^{i, j} y, \quad \varphi\left(e_{i}, x\right)=\sum_{k=1}^{s(i)} b_{k}^{i} e_{k}+b_{1,1}^{i} x+b_{2,2}^{i} y, \varphi\left(x, e_{i}\right)=\sum_{k=1}^{s(i)} d_{k}^{i} e_{k}+d_{1,1}^{i} x+d_{2,2}^{i} y, \\
& \varphi\left(e_{i}, y\right)=\sum_{k=1}^{s(i)} c_{k}^{i} e_{k}+c_{1,1}^{i} x+c_{2,2}^{i} y, \quad \varphi\left(y, e_{i}\right)=\sum_{k=1}^{s(i)} f_{k}^{i} e_{k}+f_{1,1}^{i} x+f_{2,2}^{i} y, \varphi(x, y)=\sum_{k=1}^{s} g_{k}^{i} e_{k}+g_{1,1}^{i} x+g_{2,2}^{i} y, \\
& \varphi(y, x)=\sum_{k=1}^{s} u_{k}^{i} e_{k}+u_{1,1}^{i} x+u_{2,2}^{i} y, \quad \varphi(x, x)=\sum_{k=1}^{s} v_{k}^{i} e_{k}+v_{1,1}^{i} x+v_{2,2}^{i} y, \quad \varphi(y, y)=\sum_{k=1}^{s} r_{k}^{i} e_{k}+r_{1,1}^{i} x+r_{2,2}^{i} y,
\end{aligned}
$$

where $i, j \in \mathbb{N}$.

We claim that $\varphi$ is generated by the linear function $f \in \operatorname{Hom}(R(F, 2), R(F, 2))$ given below

$$
\begin{aligned}
& f\left(e_{1}\right)=-\alpha_{1}^{1} e_{1}-\sum_{k=2}^{p_{1,1}} a_{k+1}^{1,1} e_{k}+b_{1,1}^{1} x+b_{2,2}^{1} y, \quad f\left(e_{2}\right)=d_{1}^{2} e_{1}+\sum_{k=2}^{s_{2}} \alpha_{k}^{2} e_{k}+\alpha_{1,1}^{2} x+\alpha_{2,2}^{2} y, \\
& f(x)=\tau_{1} e_{1}-\sum_{k=2}^{q} g_{k} e_{k}-b_{1}^{1} x+\tau_{2,2} y, \quad f(y)=-g_{1} e_{1}-\sum_{k=2}^{s} r_{k} e_{k}-c_{1}^{1} x+\gamma_{2,2} y, \\
& f\left(e_{i}\right)=d_{1}^{i} e_{1}+\sum_{k=2}^{s_{i}} \alpha_{k}^{i} e_{k}+a_{1,1}^{i-1,1} x+a_{2,2}^{i-1,1} y, \quad i \geq 3,
\end{aligned}
$$

that is to prove that the cocycle $\chi=\varphi-\psi \in Z^{2}(R(F, 2), R(F, 2))$, where $\psi(x, y)=f([x, y])-$ $[f(x), y]-[x, f(y)]$ is trivial. Expend the cocycle $\chi$ by the basis $\left\{x, y, e_{1}, e_{2}, \ldots\right\}$ 


$$
\begin{array}{ll}
\chi\left(e_{1}, e_{1}\right)=a_{1}^{1,1} e_{1}+a_{2}^{1,1} e_{2}+a_{1,1}^{1,1} x+a_{2,2}^{1,1} y, & \chi\left(e_{i}, y\right)=\sum_{k=1}^{s(i)} c_{k}^{i} e_{k}+c_{1,1}^{i} x+c_{2,2}^{i} y, \quad i \geq 3, \\
\chi\left(e_{1}, e_{i}\right)=\sum_{k=1}^{p(1, i)} a_{k}^{1, i} e_{k}+a_{1,1}^{1, i} x+a_{2,2}^{1, i} y, & \chi\left(e_{i}, e_{1}\right)=\sum_{k=1}^{p(i, 1)} a_{k}^{i, 1} e_{k}, \quad i \geq 2, \\
\chi\left(e_{i}, e_{j}\right)=\sum_{k=1}^{p(i, j)} a_{k}^{i, j}+a_{1,1}^{i, j} x+a_{2,2}^{i, j} y, & \chi\left(e_{i}, x\right)=\sum_{k=1}^{s_{i}} b_{k}^{i} e_{k}+b_{1,1}^{i} x+b_{2,2}^{i} y, \quad i, j \geq 2, \\
\chi\left(x, e_{i}\right)=\sum_{k=1}^{s_{i}} d_{k}^{i} e_{k}+d_{1,1}^{i} x+d_{2,2}^{i} y, & \chi\left(y, e_{i}\right)=\sum_{k=1}^{s(i)} f_{k}^{i} e_{k}+f_{1,1}^{i} x+f_{2,2}^{i} y, \quad i \geq 1, \\
\chi\left(e_{1}, y\right)=\sum_{k=2}^{s_{1}} c_{k}^{1} e_{k}+c_{1,1}^{1} x+c_{2,2}^{1} y, & \chi\left(e_{1}, x\right)=\sum_{k=2}^{s_{1}} b_{k}^{1} e_{k}, \\
\chi(y, x)=\sum_{k=1}^{s} u_{k}^{i} e_{k}+u_{1,1}^{i} x+u_{2,2}^{i} y, & \chi(x, y)=g_{1,1} x+g_{2,2} y, \\
\chi(x, x)=\sum_{k=1}^{s} v_{k}^{i} e_{k}+v_{1,1}^{i} x+v_{2,2}^{i} y, & \chi(y, y)=r_{1} e_{1}+r_{1,1}^{i} x+r_{2,2}^{i} y, \\
\chi\left(e_{2}, y\right)=c_{1}^{2} e_{1}+\sum_{k=3}^{s(i)} c_{k}^{i} e_{k}+c_{1,1}^{i} x+c_{2,2}^{i} y . &
\end{array}
$$

\begin{tabular}{|c|c|c|}
\hline$Z\left(e_{1}, e_{1}, e_{1}\right)=0$ & $\Rightarrow$ & $a_{1,1}^{1,1}=0$ \\
\hline$Z\left(e_{2}, e_{1}, e_{1}\right)=0$ & $\Rightarrow$ & $a_{1}^{1,1}=a_{2,2}^{1,1}=0$ \\
\hline$Z\left(e_{1}, e_{1}, y\right)=0, \quad i \geq 2$ & $\Rightarrow$ & $a_{2}^{1,1}=0, c_{k}^{1}=0,2 \leq k \leq s_{1}$ \\
\hline$Z\left(e_{1}, e_{1}, x\right)=0, \quad i \geq 2$ & $\Rightarrow$ & $b_{k}^{1}=0,2 \leq k \leq s_{1}$ \\
\hline$Z\left(e_{1}, x, e_{1}\right)=0$ & $\Rightarrow$ & $d_{1,1}^{1}=0$ \\
\hline$Z\left(x, e_{1}, y\right)=0$ & $\Rightarrow$ & $\begin{array}{l}g_{1,1}^{1}=c_{1,1}^{1}=c_{2,2}^{1}=0 \\
d_{k}^{1}=0, \quad 2 \leq k \leq s_{1}\end{array}$ \\
\hline$Z\left(e_{1}, e_{1}, e_{i}\right)=0, \quad i \geq 2$ & $\Rightarrow$ & $a_{k}^{1, i}=0, i \geq 2,2 \leq k \leq p(1, i)$ \\
\hline$Z\left(e_{1}, e_{i}, y\right)=0, \quad i \geq 3$ & $\Rightarrow$ & $a_{1}^{1, i}=-c_{1,1}^{i}, a_{1,1}^{1, i}=a_{2,2}^{1, i}=0, i \geq 3$ \\
\hline$Z\left(x, e_{1}, e_{i}\right)=0, \quad i \geq 2$ & $\Rightarrow$ & $d_{1,1}^{i}=d_{k}^{i}=0,2 \leq k \leq s_{i}, i \geq 2$ \\
\hline$Z\left(x, e_{i}, e_{1}\right)=0$ & $\Rightarrow$ & $\begin{array}{l}a_{1}^{2,1}=c_{1,1}^{2}=0, a_{1}^{i, 1}=c_{1,1}^{i} \\
d_{2,2}^{i}=0, i \geq 3\end{array}$ \\
\hline$Z\left(y, y, e_{i}\right)=0, \quad i \geq 1$ & $\Rightarrow$ & $r_{1,1}=0$ \\
\hline$Z\left(y, e_{i}, y\right)=0, \quad i \geq 2$ & $\Rightarrow$ & $f_{1}^{i}=f_{1,1}^{i}=f_{2,2}^{i}=0, i \geq 2$ \\
\hline$Z\left(e_{1}, x, x\right)=0$ & $\Rightarrow$ & $v_{1,1}=0$ \\
\hline$Z\left(e_{2}, x, x\right)=0$ & $\Rightarrow$ & $v_{1}=v_{2,2}=0$ \\
\hline$Z\left(x, x, e_{1}\right)=0$ & $\Rightarrow$ & $d_{1}^{1}=d_{1,1}^{1}=d_{2,2}=v_{k}=0,2 \leq k \leq s$ \\
\hline$Z\left(e_{2}, y, y\right)=0$ & $\Rightarrow$ & $r_{1}=r_{2,2}=0$ \\
\hline$Z\left(y, e_{1}, x\right)=0, \quad i \geq 2$ & $\Rightarrow$ & $u_{1,1}=u_{k}=0,2 \leq k \leq s$ \\
\hline$Z\left(e_{i}, y, e_{j}\right)=0, \quad i, j \geq 2$ & $\Rightarrow$ & $a_{1}^{i, j}=a_{1,1}^{i, j}=a_{2,2}^{i, j}=0, i, j \geq 2$ \\
\hline$Z\left(e_{1}, x, y\right)=0, \quad i \geq 2$ & $\Rightarrow$ & $g_{1,1}=0$ \\
\hline$Z\left(x, e_{i}, y\right)=0, \quad i \geq 2$ & $\Rightarrow$ & $c_{1}^{i}=d_{2,2}^{i}=0, i \geq 2$ \\
\hline$Z\left(e_{1}, e_{2}, y\right)=0$ & $\Rightarrow$ & $a_{1,1}^{2}=a_{2,2}^{2}=c_{1,1}^{2}=0$ \\
\hline
\end{tabular}

Now, we impose the cocycle identities $\mathrm{Z}=0$ (see 2.2) to $\chi$ and derive a set of constraints for the coefficients as follows. 


$\begin{array}{ll}\text { 2-cocyle identity } & \text { Constraints } \\ Z\left(e_{2}, e_{1}, y\right)=0, & \Rightarrow\left\{\begin{array}{l}c_{2}^{3}=0, c_{k}^{i}=c_{k-1}^{2}, 3 \leq k \leq s_{2}+1, \\ a_{k}^{i, j}=0,2 \leq k \leq p_{i, j}, i, j \geq 2,\end{array}\right. \\ Z\left(e_{i}, e_{j}, y\right)=0, \quad i \geq 2, \quad \Rightarrow\left\{\begin{array}{l}c_{k}^{2}=0,3 \leq k \leq s_{2}, \\ b_{1}^{i}=b_{1,1}^{i}=b_{2,2}^{i}=0, i \geq 2, \\ Z\left(e_{i}, x, y\right)=0, i \geq 2,\end{array}\right. \\ Z\left(e_{2}, y, x\right)=0,\end{array} \quad \Rightarrow\left\{\begin{array}{l}u_{1}=u_{2,2}=0,\end{array}\right.$

As a result we get

$$
\chi\left(e_{i}, e_{1}\right)=\sum_{k=2}^{p_{i, 1}} a_{k}^{i, 1} e_{k}, \quad \chi\left(e_{i}, x\right)=\sum_{k=2}^{s_{i}} b_{k}^{i} e_{k}, i \geq 2 .
$$

Observe that we have not imposed any constraints for the components $\alpha_{k}^{i}, 2 \leq i, 2 \leq k \leq s_{i}$ of $f\left(e_{i}\right)$ in (3.4) yet. Now choosing the coefficients $\alpha_{k}^{i}, 2 \leq i, 2 \leq k \leq s_{i}$ in (3.4) we attain $\chi\left(e_{i}, e_{1}\right)=0$ for $i \geq 2$. Since, $\chi\left(e_{i}, x\right)$ are expressed via $\chi\left(e_{i}, e_{1}\right)$, from $Z\left(e_{i}, e_{1}, x\right)=0, i \geq 2$, we conclude that $\chi\left(e_{i}, x\right)=0, i \geq 2$. This completes the proof.

Acknowledgements. The authors are grateful to the referee for valuable comments and remarks.

\section{REFERENCES}

[1] K.K.Abdurasulov, Maximal solvable extension of nilpotent Leibniz algebras, DAN RUz, 2019, vol.5, 3-8.

[2] K.K. Abdurasulov, Kh.A. Khalkulova, Solvable Lie algebras with maximal dimension of complementary space to nilradical, Uzbek Mathematical Journal, 2018, vol.1, 90-98.

[3] K.K. Abdurasulov, G.O. Solijanova, Maximal pro-solvable Lie algebras with maximal positively graded ideals of length $\frac{3}{2}$, Bulletin of the Institute of Mathematics, 2020, vol.5, 25-32.

[4] J.M. Ancochea Bermudez, R. Campoamor-Stursberg, Cohomologically rigid solvable Lie algebras with a nilradical of arbitrary characteristic sequence, Linear Algebra and Its Applications, 2016, 488, 135-147.

[5] D.W. Barnes, On Levi's theorem for Leibniz algebras, Bull. Aust. Math. Soc., 2012, 86(2), 184-185.

[6] L. Bosko-Dunbar, J.D. Dunbar, J.T. Hird, K. Stagg, Solvable Leibniz algebras with Heisenberg nilradical, Communications in Algebra, 2015, 43(6), 2272-2281.

[7] J.M. Casas, M. Ladra, B.A. Omirov, I. Karimjanov, A Classification of solvable Leibniz algebras with null-filiform nilradical, Linear and Multilinear Algebra, 2013, 61(6), 758-774.

[8] J.M. Casas, M. Ladra, B.A. Omirov, I. Karimjanov, Classification of solvable Leibniz algebras with naturally graded fliform nilradical, Linear Algebra and its Applications, 2013, 438(7), 2973-3000.

[9] R. Campoamor-Stursberg, Solvable Lie algebras with an $\mathbb{N}$-graded nilradical of maximum nilpotency degree and their invariants, J. Physics A., 2010, 43(14), 145-202.

[10] E.M. Cañete, A.Kh. Khudoyberdiyev, The classification of 4-dimensional Leibniz algebras, Linear Algebra and its Applications, 2013, 439, 273-288.

[11] A. Fialowski, Classification of Graded Lie Algebras with Two Generators, Vestn. Mosk. Univ., Ser. 1: Mat., Mekh., 1983, 2, 62-64 [Moscow Univ. Math. Bull., 1983, 38(2), 76-79].

[12] R.K. Gaybullaev, Outer derivations of complex solvable Lie algebras with nilradical of maximal rank, Uzbek Mathematical Journal, 2020, 4, 40-43.

[13] R.K. Gaybullaev, A.Kh. Khudoyberdiyev, K. Pohl, Classification of solvable Leibniz algebras with abelian nilradical and $(k-1)$-dimensional extension, Communications in Algebra, 2020, 48(7), 3061-3078.

[14] I.A. Karimjanov, A.Kh. Khudoyberdiyev, B.A. Omirov, Solvable Leibniz algebras with triangular nilradicals, Linear Algebra and its Applications, 2015, 466, 530-546.

[15] K. Khakimdjanova, Yu. Khakimdjanov, Sur une classe d'algebres de Lie de dimension infinie, Communications in Algebra, 2001, 29(1), 177-191 (in French).

[16] K.K. Kudoybergenov, M. Ladra, B.A. Omirov, On Levi-Malcev theorem for Leibniz algebras, Linear and Multilinear Algebra, 2019, 67(7), 1471-1482.

[17] A.Kh. Khudoyberdiyev, M. Ladra, B.A. Omirov, On solvable Leibniz algebras whose nilradical is a direct sum of null-filiform algebras, Linear and Multilinear Algebra, 2014, 62(9), 1220-1239.

[18] A.Kh. Khudoyberdiyev, I.S. Rakhimov, Sh.K. Said Husain, On classification of 5-dimensional solvable Leibniz algebras, Linear Algebra and its Applications, 2014, 457, 428-454.

[19] M. Ladra, K.K. Masutova, B.A. Omirov, Corrigendum to "Classification of solvable Leibniz algebras with naturally graded filiform nilradical" [Linear Alg. Appl., 2013, 438(7), 2973-3000], Linear Alg. Appl., 2016, 507, 513-517.

[20] G. Leger, E. Luks, Cohomology theorems for Borel-like solvable Lie algebras in arbitrary characteristic, Canadian J. Math., 1972, 24, 1019-1026.

[21] U.X. Mamadaliyev, A rigid solvable Leibniz algebra, Uzbek Mathematical Journal, 2013, 3, 70-78. (in Russian)

[22] D. V. Millionshchikov, Naturally graded Lie algebras of slow growth, Mat. Sb., 2019, 210(6), 111-160. (in Russian)

[23] D.V. Millionschikov, Cohomology of graded Lie algebras of maximal class with coefficients in the adjoint representation, Proceedings of the Steklov Institute of Mathematics, 2008, 263, 99-111. 
[24] V.V. Morozov, Classification of nilpotent Lie algebras of sixth order, Izv. Vyš̌. Učebn. Zaved. Matematika, 1958, 5(4), 161-171 (in Russian).

[25] G.M. Mubarakzjanov, On solvable Lie algebras, Izv. Vyš̌. Učebn. Zaved. Matematika, 1963, 32(1), $114-123$ (in Russian)

[26] G.M. Mubarakzjanov, Classification of real structures of Lie algebras of fifth order, Izv. Vysš. Učebn. Zaved. Matematika, 1963, 34(3), 99-106 (in Russian).

[27] G.M. Mubarakzjanov, Classification of solvable Lie algebras of sixth order with a non-nilpotent basis element, Izv. Vyš̌. Učebn. Zaved. Matematika, 1963, 35(4), 104-116 (in Russian).

[28] G.M. Mubarakzjanov, Certain theorems on solvable Lie algebras, Izv. Vyš̌. Učebn. Zaved. Matematika, 1966, 55(6), 95-98 (in Russian).

[29] J.-C. Ndogmo and P. Winternitz, Generalized Casimir operators of solvable Lie algebras with abelian nilradicals, J. Phys., 1994, A 27(8), 2787-2800.

[30] J.-C. Ndogmo and P. Winternitz, Solvable Lie algebras with abelian nilradicals, J. Phys., 1994, A 27(2), 405-423.

[31] B.A. Omirov, Thin Leibniz Algebras, Mathematical Notes, Vol. 80 No. 2 (2006), 244-253.

[32] L.M. Camacho, B.A. Omirov, K.K. Masutova, Solvable Leibniz algebras with filiform nilradical, Bull. Malays. Math. Sci. Soc., 2016, 39(1), 283-303.

[33] J. L. Rubin and P. Winternitz, Solvable Lie algebras with Heisenberg ideals, J. Phys., A 26(5), (1993), 1123-1138.

[34] A. Shabanskaya, Solvable indecomposable extensions of two nilpotent Lie algebras, Communications in Algebra, 2016, 44(8), 3626-3667.

[35] A. Shabanskaya, G. Thompson, Six-dimensional Lie algebras with a five-dimensional nilradical, J. Lie Theory, 2013, 23(2), 313-355.

[36] A. Shabanskaya, G. Thompson, Solvable extensions of a special class of nilpotent Lie algebras, Arch. Math., (Brno), 2013, 49(3), 63-81.

[37] A. Shabanskaya, Right and left solvable extensions of an associative Leibniz algebra, Communications in Algebra, 2017, 45(6), 2633-2661.

[38] S̆nobl and P. Winternitz. Classification and Identification of Lie Algebras, CRM Monograph Series, AMS, 2014 33, 306 pp.

[39] L. S̆nobl and P. Winternitz, A class of solvable Lie algebras and their Casimir invariants, J. Phys., 2005, A 38(12), $2687-2700$

[40] L. S̆nobl and P. Winternitz, All solvable extensions of a class of nilpotent Lie algebras of dimension $n$ and degree of nilpotency $n-1$, J. Phys., 2009, A 42(10), 105201.

[41] L. Šnobl and P. Winternitz, Solvable Lie algebras with Borel nilradicals, J. Phys., 2012, A 45(9), 095202.

[42] B.Ju. Weisfeiler, Infinite-dimensional filtered Lie algebras and their connection with graded Lie algebras, Funkt. Analis $i$ ego Prilozh., 1968, 2(1), 94-95. (English translation: Funct. Anal. Appl., 1968, 2, 88-89.)

[43] M. Vergne, Cohomologie des algébres de Lie nilpotentes. Application á l'étude de la variété des algébres de Lie nilpotentes, Bull. Soc. Math. France, 1970, 98, 81-116 (in French).

[44] E.I. Zel'manov, On some problems of group theory and Lie algebras, Mathematics of the USSR-Sbornik, 1990, 66(1), 159-168.

${ }^{1}$ Institute of Mathematics Uzbekistan Academy of Sciences, Tashkent, Uzbekistan

Email address: abdurasulov0505@mail.ru, omirovb@mail.ru

2 Faculty of Computer and Mathematical Sciences, Universiti Teknologi Mara (Uitm), Shah Alam, MALAYSIA

Email address: isamiddin@uitm.edu.my

3 National University of Uzbekistan, Tashkent, Uzbekistan

Email address: gulhayo.solijonova@mail.ru 